\title{
Mesa Redonda de presentación del Movimiento Sanitario Argentino (MOSA)
}

\section{Round table for the presentation of the Argentinean Health Movement (MOSA)}

\section{Dr. J osé Carlos Escudero}

Universidad de Luján. Buenos Aires, Argentina.

La actividad se realizó en la Facultad de Ciencias Sociales de la Universidad de Buenos Aires, el 14 de noviembre de 2011.

Compañeros y compañeras: mi primer comentario es que los que trabajamos el tema de la "salud" llevamos una pesada mochila: nos ocupamos de la vida y de la muerte. Si trabajamos el tema de la "comunicación" podemos ocuparnos de los satélites, de la bandas de frecuencia, de la digitalización, temas de tecnología. Salud es cualitativamente distinta, sus fallas extremas se traducen en muertes que podían no haber ocurrido, y que suceden las $24 \mathrm{~h}$ del día, todos los días. Llevamos además, en la Argentina $\mathrm{K}$ de hoy otra mochila, que esperamos sea solamente coyuntural: yo tengo cierta edad más o menos avanzada, y hasta hace poco pensaba que me iba a morir sin llegar a ver en nuestro país cosas que deseaba y que están en el centro de mi vida. Muchas de esas cosas que yo considero maravillosas- han ocurrido o están tendencialmente ocurriendo en nuestro país desde el 2003, con la llegada al gobierno de los compañeros Nestor y Cristina... pero lo que me pregunto, y creo que muchos entre ustedes se preguntan, por qué estas cosas maravillosas no están sucediendo en el sector de la salud, mejorando nuestra salud colectiva. Uno hubiera pensado que, por ejemplo, en estos años la oferta estatal gratuita de salud hubiera aumentado significativamente en efectores públicos de salud, y con prestaciones provistas por funcionarios estatales con trabajo en blanco e integrantes del Servicio Civil del 
Estado. Nada de esto sucedió, y no es porque el Estado nacional no tuviera dinero. Lo tiene, a una escala mucho mayor que hace diez años, gracias sobretodo a las maravillosas macropolíticas económicas kircheneristas, con algunos detalles menores adicionales: sin modificar de raíz el sistema impositivo nacional, solamente con apretar las clavijas al existente, y con el círculo virtuoso keynesiano en funcionamiento, el peso impositivo del Estado en el PBI nacional aumentó -creo recordar- siete puntos. Hay plata, hay un montón de plata que parece inconcebible para quienes mirábamos la cosa hace diez años. Asociado con esto, insistamos que, para el sector de la salud, hay que terminar con los financiamientos que tan generosamente nos proponen los organismos internacionales de crédito y que tan emprendedoramente aceptan nuestros funcionarios sanitarios. Estos programas aumentan el endeudamiento externo, son puntuales y recortados, tienen altos gastos administrativos, están sobrepoblados por asesores, consultores, consultoras; y están programados para que los funcionarios argentinos viajen mucho, sobre todo a Washington. Pareciera que ciertos funcionarios sanitarios tienen debilidad por estos viajes que aparentemente los sensibilizan a aceptar el paquete de los programas que se les ofrecen allí. Esta es una pequeña corrupción que, junto con otras corrupciones mucho mas grandes, aceita los engranajes de las decisiones públicas y privadas en el sector de la salud. No debería haber financiaciones internacionales para nuestra salud estatal, todo el gasto debe efectuarse con recursos del Estado argentino.

Unas reflexiones sobre Perón, Carrillo y la salud en el primer peronismo. Perón ganó las elecciones presidenciales de 1946, luego de 18 años con elecciones fraudulentas que imposibilitaban que el radicalismo partido mayoritario pudiera ganar. En 1946 no había encuestas de intenciones de voto, ni bocas de urna, ni escrutinios inmediatos en las mesas de votación. Se fue escrutando de a poco, los primeros resultados favorecían a la Unión Democrática, que unificaba a la oposición, a la cual pertenecían, recordemos, partidos autocalificados de "progres", como el Socialista, Comunista, Demócrata Progresista, más las fracciones de izquierda llamémoslas populistas- del radicalismo, como el "sabatinismo". Cuando se escrutó toda la votación, resultó que Perón había ganado, para estupefacción de la oposición, que no se animó a llamar fraudulentas a las elecciones más limpias de la historia argentina hasta ese momento. Perón le dio toda su confianza y muchísimo dinero a Carrillo, que no era sanitarista, pero que con poder e inteligencia para usarlo, gastó ese dinero de la manera más rápida y eficiente: aumentando enormemente la oferta pública de salud gratuita, en un sistema de ofertores estatales a los que se accedía por derecho de ciudadanía; lo que maximizaba la eficiencia, en parte por economías de escala. Esto se hizo en el mundo un par de años antes de la implementación del National Health Service (NHS) de Gran Bretaña, ese otro enclave socialista de salud en un contexto capitalista, que al día de hoy casi monopoliza este tema en los escritos de la Academia internacional. Perón aprovechó el desconcierto de la oposición, que perdió mientras creía que ganaba, y aprovechó a Carrillo, que combinaba una cultura y una capacidad de trabajo excepcionales con una honradez extrema, que lo llevó a la muerte en la miseria cuando tuvo que exiliarse. Vinculado con todo esto, debemos reflexionar muy seriamente hoy para calibrar la situación argentina, mundial y a nuestros enemigos actuales, que en el año 1946 tenían menos capacidad de daño que ahora: no había entonces organismos internacionales de crédito, el capitalismo no veía en la salud a un área para invertir, las intervenciones desestabilizadoras de EE. UU. no habían empezado (en América Latina, la primera, en Guatemala, fue en 1954), y los Medios internacionales no eran tan monolíticamente voceros de las necesidades capitalistas y mentirosos como hoy.

Una reflexión sobre la actual Europa ajustadora y neoliberal. ¿Se acuerdan cuando la sabiduría convencional calificaba de "serios" a los países europeos, diferenciándolos de nuestra "poca seriedad" argentina? Esta visión está en el ADN de la clase media argentina, especialmente la porteña y la descendiente de inmigrantes europeos. Lo que está sucediendo ahora en Europa es una crisis tremenda, cuyo sufrimiento 
humano está apenas empezando. El desempleo y la precarización laboral aumentan, las certezas se vuelven incertidumbres, los hijos dejan de tener un futuro mejor que los padres, su nivel educativo baja, la salud colectiva buena y segura se vuelve cara y de difícil acceso. Pensemos en las autoagresiones, las agresiones, los insomnios, las enfermedades psicosomáticas que aumentan, la salud colectiva preventiva que se dificulta. En todos lados hay crecientes restricciones para lograr la salud que daban los sistemas universales y gratuitos. En todos los países europeos las distancias entre ricos y pobres están aumentando. Esto es un acicate adicional para que los cada vez más escasos ricos afortunados compren seguros privados de salud, para llenar el vacío que dejan los sistemas universales, desfinanciados y saboteados, que se retiran.

Una reflexión sobre nuestros enemigos. No nos engañemos. Si uno es capitalista, gana más plata invirtiendo en salud que invirtiendo en cualquier otra área de la economía, y es casi imposible auditar la forma en que se gasta el dinero en el sector. En estos días, por primera vez en la historia, los salarios y beneficios de los empresarios de salud en EE. UU. ha llegado a ser superior a los de los banqueros de Wall Street. Esto ayuda a explicar el alto costo y la gran ineficiencia de la salud colectiva de ese país. Nuestro Estado argentino debe no solo regular lo que sucede en el sector sino también ser el efector predominante en nuestra salud, lo cual además minimiza la corrupción. En salud capitalista hay vía libre para que en medicamentos se vendan muchos productos innecesarios, independientemente de su iatrogenia. Además, el capitalismo crea subjetividad a través de sus Medios, que aprovechan la ansiedad sobre la salud individual de la población. Todo esto pronostica que, si queremos meter justicia social en la salud colectiva argentina, el listado de nuestros enemigos va a ser mucho más largo que el que Perón y Carrillo tuvieron que enfrentar en 1946.

Debemos prever todo esto cuando empiecen a aparecer las medidas importantes. Debemos publicitarlas, trabajar en la organización de un poder territorial que las apoye - salud se presta mucho para esto- y apoye al gobierno que las toma. Afortunadamente, salud crea una legitimidad mucho más rápidamente que, por ejemplo, educación o vivienda. Debemos saber que el menor de los errores o lo que puede ser vendido a la población como error- va a ser publicitado al máximo en los Medios. En cuanto a la situación general, y a la correlación desfavorable de fuerzas para el campo popular en el sector, hay que insistir: tengamos la cabeza fría, hagamos realpolitik en el buen sentido. Debemos pensar donde está la contradicción principal, donde la secundaria, y donde no hay contradicción sino diferentes cronologías para tomar ciertas medidas. Que quede claro que la historia argentina no es la de Suecia, Cuba, Chile, Uruguay o EE. UU. Tenemos particularidades: que los dos movimientos populares, cuya acción potencial era mucho más amenazadora para los intereses de los dueños del país que la verba de minorías esclarecidas, fueron proscriptos por 18 años cada uno. Que una rama de las Fuerzas Armadas nacionales asesinó, por bombardeo aéreo sin aviso a la plaza más importante de la capital del país, a más de 300 ciudadanos totalmente desprevenidos. La excusa que se ha venido diciendo hasta hoy - un magnicidio para librar el país de un tirano sanguinario- es falsa, no había la menor posibilidad de matar a Perón con los aviones, las bombas y las espoletas que se usaron. Se trataba de asesinar gente al descubierto para imponer el terror, de manera de no tener movilizaciones en la calle. Esta lección con baño de sangre incluido fue un antecedente para el golpe militar que tres meses después derrocó a un gobierno democrático y e impuso una proscripción de 18 años.

En la realpolitik a aplicar en la salud colectiva argentina está la problemática de las Obras Sociales. En Argentina estas se constituyeron de abajo para arriba, en el resto de Latinoamérica se hicieron de arriba para abajo, para aumentar la legitimidad de los gobiernos en la clase media, diferenciando a la salud colectiva (especialmente la atención) de la de los pobres. Los pobres de Argentina ya estaban contenidos por el excelente modelo Carrillo y esta búsqueda de legitimidad ya estaba asegurada. 
Teniendo en cuenta esta historia, toda reforma en las obras sociales, que son centrales en la construcción y defensa de un proyecto nacional y popular, debe planificarse con suma cautela, con medidas puntuales para eliminar la corrupción y para cortar el flujo de recursos generados por toda la sociedad y por los trabajadores que se está canalizando hacia la salud privada y hacia los medicamentos y las tecnologías ofrecidos desde el lucro. Voy a plantear donde creo que en el año 2012 y siguientes se plantea la principal contradicción que hay que vencer para que tengamos una salud colectiva más eficiente, inclusora y equitativa: los medicamentos. Estamos gastando en ellos un tercio del gasto total en salud. Una suma que, ante ciertos escenarios de poder, podría reducirse a una pequeña fracción, reasignando el dinero ahorrado donde es realmente eficaz. En los últimos tiempos hubo desarrollos alentadores que han debilitado a este enemigo estructural de una buena salud colectiva, la fabricación comercial de medicamentos. A nivel internacional, se nota una creciente fragmentación del bloque hegemónico, entre fabricantes multinacionales, fabricantes nacionales, fabricantes de genéricos y fabricación dentro de los Estados nacionales. Este último grupo es especialmente pujante en Argentina, y la reciente sanción de una ley de fabricación pública lo ha fortalecido. Pueden ofrecer medicamentos esenciales y otros, a un precio más bajo y con una calidad promedial mayor que los de la industria comercial. Además, pueden mostrar con su ejemplo la existencia al interior de la industria comercial, de repetidas violaciones a la validación científica de las drogas, y de repetidas violaciones a la ética de la Investigación Científica; ambas efectuadas con el fin de ganar más dinero. Que podamos denunciar estos delitos con ejemplos, y que podamos demostrar que el Estado no los comete, es una forma de legitimación de nuestro proyecto nacional y popular ante personas que por ahí no lo comparten, pero que toman en serio a la ciencia y a la ética, y que pueden convertirse en nuestros aliados.

Recibido: 16 de enero de 2012.

Aprobado: 25 de abril de 2012.

José Carlos Escudero. Universidad de Lujan. Buenos Aires, Argentina. 